\title{
THE CESÀRO OPERATOR ON DUALS OF SMOOTH SEQUENCE SPACES OF INFINITE TYPE
}

\author{
ERSIN KIZGUT
}

\begin{abstract}
The discrete Cesàro operator $C$ is investigated in strong duals of smooth sequence spaces of infinite type. Of main interest is its spectrum, which turns out to be distinctly different in the cases when the space is nuclear and when it is not.
\end{abstract}

\section{INTRODUCTION}

The discrete Cesàro operator $\mathrm{C}$ acting on $\mathbb{C}^{\mathbb{N}}$ is defined by

$$
\mathrm{C} x:=\left(x_{1}, \frac{x_{1}+x_{2}}{2}, \frac{x_{1}+x_{2}+x_{3}}{3}, \ldots, \frac{x_{1}+\cdots+x_{i}}{i}, \ldots\right), x=\left(x_{i}\right)_{i \in \mathbb{N}},
$$

which is a bicontinuous isomorphism of $\mathbb{C}^{\mathbb{N}}$ onto itself with

$$
\mathrm{C}^{-1} y=\left(i y_{i}-(i-1) y_{i-1}\right), \quad y=\left(y_{i}\right) \in \mathbb{C}^{\mathbb{N}} .
$$

For a diverse family of classical Banach spaces, the fundamental questions of continuity and determination of the spectrum have been investigated, and precise answers have been obtained. We refer the reader to the introduction of [3]. The behaviour of $\mathrm{C}$ when acting on the Fréchet spaces $\mathbb{C}^{\mathbb{N}}, \ell_{p+}=\bigcap_{q>p} \ell_{q}, 1 \leq p<\infty$, and on the strong duals $\left(\Lambda_{\infty}(\alpha)\right)_{b}^{\prime}$ of power series space of infinite type were studied in $[2,4,5]$. In this paper we generalize the results of $[5]$ to the setting of the duals of certain types of Köthe echelon spaces, so called smooth sequence spaces of infinite type. These spaces were introduced by Terzioğlu $[21,22,23,18]$. We also refer to Kocatepe $[11,12,13]$. The aim of this paper is to investigate the behaviour of $\mathrm{C}$ when it acts on the strong duals $\left(\lambda_{1}(A)\right)_{b}^{\prime}$ of smooth sequence spaces of infinite type. The reason for focusing on the infinite type dual spaces is that the Cesàro operator $C$ fails to be continuous on most of the finite type dual spaces (see Proposition 2.5). Some of our proofs are inspired by [5], but new arguments are needed in this setting. We distinctly expain our context. Let $A=\left(a_{n}\right)_{n}$, where $a_{n}=\left(a_{n}(i)\right)_{i}$. $A$ is called a Köthe matrix if the following conditions are satisfied:

(K1) $0 \leq a_{n}(i) \leq a_{n+1}(i)$, for all $i, n \in \mathbb{N}$.

(K2) For all $i \in \mathbb{N}$, there exists $n \in \mathbb{N}$ such that $a_{n}(i)>0$.

The Köthe echelon space $\lambda_{1}(A)$ of order 1 is defined by

$$
\lambda_{1}(A):=\left\{x \in \mathbb{C}^{\mathbb{N}}: \sum_{i=1}^{\infty} a_{n}(i)\left|x_{i}\right|<\infty, \forall n \in \mathbb{N}\right\}
$$

2010 Mathematics Subject Classification. 47A10, 47B37, 46A45, 46A04.

Key words and phrases. Cesàro operator, duals of smooth sequence spaces, generalized power series spaces, spectrum, (LB)-space. 
which is a Fréchet space when equipped with the increasing system of seminorms

$$
p_{n}(x)=\sum_{i=1}^{\infty} a_{n}(i)\left|x_{i}\right|, \quad x \in \lambda_{1}(A), n \in \mathbb{N} .
$$

Then $\lambda_{1}(A)=\bigcap_{n \in \mathbb{N}} \ell_{1}\left(a_{n}\right)$, where $\ell_{1}\left(a_{n}\right)$ is the usual Banach space. The space $\lambda_{1}(A)$ is given by the projective limit topology, that is, $\lambda_{1}(A)=\operatorname{proj}_{n} \ell_{1}\left(a_{n}\right)$. For the theory of Köthe echelon spaces $\lambda_{p}(A)$ of order $p$ for $1 \leq p \leq \infty$ or $p=0$, see [16, Section 27]. Let $V=\left(v_{n}\right)_{n}=\left(\frac{1}{a_{n}}\right)_{n}$. Then, the corresponding co-echelon space of $\lambda_{1}(A)$ is given by the (LB)-space $k_{\infty}(V):=\operatorname{ind}_{n} \ell_{\infty}\left(v_{n}\right)$. For co-echelon spaces, the reader is referred to $[6,7,14,16]$. A Köthe echelon space $\lambda_{1}(A)$ is said to be a smooth sequence space of infinite type (or a $G_{\infty}$-space) [21, Section 3] if $A$ satisfies $(\mathrm{G} \infty-1) 1 \leq a_{n}(i) \leq a_{n}(i+1)$, for all $i, n \in \mathbb{N}$.

$(\mathrm{G} \infty-2)$ For all $n \in \mathbb{N}$ there exist $m>n$ and $M>0$ such that $a_{n}(i)^{2} \leq M a_{m}(i)$, for all $i \in \mathbb{N}$.

Proposition 1.1. [21, 3.1][7, Theorem 4.9] For a $G_{\infty}$-Köthe matrix A, the following statements are equivalent:

(1) $\lambda_{1}(A)$ is a Schwartz space.

(2) $\lambda_{1}(A)$ is not isomorphic to $\ell_{1}$.

(3) There exists $n \in \mathbb{N}$ such that

$$
\lim _{i \rightarrow \infty} a_{n}(i)=\infty
$$

(4) For all $n \in \mathbb{N}$ there exists $m>n$ such that

$$
\lim _{i \rightarrow \infty} \frac{v_{m}(i)}{v_{n}(i)}=\lim _{i \rightarrow \infty} \frac{a_{n}(i)}{a_{m}(i)}=0 .
$$

(5) $k_{\infty}(V)$ is isomorphic to $k_{0}(V)=\operatorname{ind}_{n} c_{0}\left(v_{n}\right)$.

(6) $k_{0}(V)$ is a Montel space.

In the light of Proposition 1.1, we deal with the Cesàro operator $\mathrm{C}$ defined on the co-echelon space $k_{0}(V)$ of order 0 . Indeed, since the Köthe echelon space $\lambda_{1}(A)$ of order 1 is a Fréchet-Schwartz space (hence, distinguished) in our case, it follows that $k_{0}(V)=\operatorname{ind}_{n} c_{0}\left(v_{n}\right)=\operatorname{ind}_{n} \ell_{\infty}\left(v_{n}\right)=\left(\lambda_{1}(A)\right)_{b}^{\prime}$ is the strong dual of $\lambda_{1}(A)$. For each $n \in \mathbb{N}$ we define the norm

$$
q_{n}(x):=\sup _{i \in \mathbb{N}} v_{n}(i)\left|x_{i}\right|, \quad x=\left(x_{i}\right) \in \ell_{\infty}\left(v_{n}\right)
$$

whose restriction to $c_{0}\left(v_{n}\right)$ is the norm in $c_{0}\left(v_{n}\right)$. For each $n \in \mathbb{N}, c_{0}\left(v_{n}\right) \subseteq c_{0}\left(v_{m}\right)$, for every $m \geq n$ and

$$
q_{m}(x) \leq q_{n}(x), \quad \forall x \in c_{0}\left(v_{n}\right) .
$$

Let us remind that nuclear spaces are in particular Schwartz. Since $k_{0}(V)=$ $\left(\lambda_{1}(A)\right)_{b}^{\prime}$, the nuclearity of $k_{0}(V)$ is equivalent to that of $\lambda_{1}(A)$. The following result is known (see $[21,3.1-\mathrm{b}]$ ), however, we give a partial proof.

Proposition 1.2. For a $G_{\infty}$-Köthe matrix $A$, the following statements are equivalent:

(1) For any $n \in \mathbb{N}$ there exists $m>n$ such that

$$
\sum_{i=1}^{\infty} \frac{v_{m}(i)}{v_{n}(i)}=\sum_{i=1}^{\infty} \frac{a_{n}(i)}{a_{m}(i)}<\infty .
$$


(2) $\lambda_{1}(A)$ is nuclear.

(3) $k_{0}(V)$ is nuclear.

(4) There exists $n \in \mathbb{N}$ such that

$$
\sum_{i=1}^{\infty} v_{n}(i)<\infty
$$

Proof. $(1) \Leftrightarrow(2)$ See e.g. [16, Proposition 28.15].

$(2) \Leftrightarrow(3)$ Follows by [17, pp.78].

$(3) \Rightarrow(4)$ Since $\lambda_{1}(A)$ is a Schwartz space, by Proposition 1.1, we may pick $n \in \mathbb{N}$ as in (I). So there exists $M>0$ with $v_{n}(i) \leq M$, for all $i \in \mathbb{N}$. Since $k_{0}(V)$ is nuclear, we may choose an $m>n$ as in (GPC). Hence

$$
\sum_{i=1}^{\infty} v_{m}(i)=\sum_{i=1}^{\infty} v_{n}(i) \frac{v_{m}(i)}{v_{n}(i)} \leq M \sum_{i=1}^{\infty} \frac{v_{m}(i)}{v_{n}(i)}<\infty .
$$

(4) $\Rightarrow(3)$ Suppose that there exists $n_{0}$ as in (SV). For an $n \geq n_{0}$, if we pick $m>n$ and $C>0$ as in $(\mathrm{G} \infty-2)$, then

$$
\sum_{i=1}^{\infty} \frac{v_{m}(i)}{v_{n}(i)} \leq C \sum_{i=1}^{\infty} v_{n}(i)<\infty
$$

since $(\mathrm{G} \infty-1)$ implies that $v_{n}(i)<v_{n_{0}}(i)$, for all $i \in \mathbb{N}$. That means $(\mathrm{GPC})$ is satisfied, and so $k_{0}(V)$ is nuclear.

A power series space $\Lambda_{\infty}^{1}(\alpha)=\left\{x \in \mathbb{C}^{\mathbb{N}}\left|\sum_{i=1}^{\infty} \exp \left(\alpha_{i} n\right)\right| x_{i} \mid<\infty, \forall n \in \mathbb{N}\right\}$ of infinite type associated with the strictly increasing sequence $\alpha_{i} \stackrel{i}{\rightarrow} \infty$ is a $G_{\infty}$-space (see [16, Section 29] for power series spaces of infinite type). But the converse is false, in general as shown in Example 1.3 below. A Fréchet space $E$ equipped with the increasing system $\left(p_{n}(\cdot)\right)_{n \in \mathbb{N}}$ of seminorms is said to have property (DN) [16, pp. 359] if there exists $s \in \mathbb{N}$ such that for all $n \in \mathbb{N}$ there exist $m \in \mathbb{N}$ and $C>0$ satisfying

$$
p_{n}(x)^{2} \leq C p_{m}(x) p_{s}(x), \quad \forall x \in E .
$$

Here, such $p_{s}(\cdot)$ is a norm and is called the dominating norm. It is straightforward to prove that a power series space $\Lambda_{\infty}^{1}(\alpha)$ of infinite type satisfies property (DN). Example 1.3 also illustrates that a $G_{\infty}$-space satisfying condition (DN) is still not necessarily isomorphic to a power series space of infinite type.

A Dragilev space of infinite type $L_{f}\left(\alpha_{i}, \infty\right)$ is defined via $f: \mathbb{R} \rightarrow \mathbb{R}^{+}$an odd, increasing, logarithmically convex (i.e., $\log \circ f$ is convex function for $x>0$ ), and the strictly increasing sequence $\alpha_{i} \stackrel{i}{\rightarrow} \infty$. If $a_{n}(i)=\exp \left(f\left(n \alpha_{i}\right)\right)$, then $L_{f}\left(\alpha_{i}, \infty\right)$ is isomorphic to the Köthe echelon space $\lambda_{1}(A)$ of order 1 . Let $0<\rho<\infty$, then the limit $\tau(\rho)=\lim _{x \rightarrow \infty} \frac{f(\rho x)}{f(x)} \leq \infty$ exists. The function $f$ is called rapidly increasing if $\tau(\rho)=\infty$, for all $\rho>1$. Otherwise, $f$ is called slowly increasing. In [9, Section 3.2 ], it is explained that the space $L_{f}\left(\alpha_{i}, \infty\right)$ is isomorphic to a power series space of infinite type if and only if $f$ is slowly increasing.

Example 1.3. Let $A$ be an infinite matrix defined by $a_{n}(i):=\exp \left(i n e^{i n}\right)$. Then, the space $X:=\left\{x \in \mathbb{C}^{\mathbb{N}}: \sum_{i=1}^{\infty} a_{n}(i)\left|x_{i}\right|<\infty, \forall n \in \mathbb{N}\right\}$, is a nuclear $G_{\infty}$-space satisfying property (DN) which is not isomorphic to a power series space of infinite type. 
Proof. (i) $X$ is a nuclear $G_{\infty}$-space: It is trivial to check that for any $n \in \mathbb{N}$ one has $0<a_{n}(i) \leq a_{m}(i)$, for all $i \in \mathbb{N}$ and for all $m \geq n$, so $X$ is a Köthe echelon space. It is also clear that for all $i \in \mathbb{N}$ we have $1 \leq a_{n}(i) \leq a_{n}(i+1)$ so $(\mathrm{G} \infty-1)$ is satisfied. Now given $n \in \mathbb{N}$, choose $m=2 n$. Then $a_{n}(i)^{2}=\exp \left(2\right.$ ine $\left.e^{i n}\right) \leq$ $\exp \left(2 i n e^{2 i n}\right)=a_{m}(i)$ holds for all $i, n \in \mathbb{N}$. So $\left(\mathrm{G}_{\infty}-2\right)$ is also satisfied. Hence $X$ is a $G_{\infty}$-space. For nuclearity, consider

$$
\log (i)<i n \Rightarrow i<e^{i n}<n e^{i n} \Rightarrow-n e^{i n}<-i .
$$

Given $n \in \mathbb{N}$, select $m>n$ and $M>0$ as in $(\mathrm{G} \infty-2)$. Then,

$$
\sum_{i=1}^{\infty} \frac{a_{n}(i)}{a_{m}(i)} \leq \sum_{i=1}^{\infty} M \frac{a_{n}(i)}{a_{n}(i)^{2}}=M \sum_{i=1}^{\infty} \frac{1}{a_{n}(i)} \leq M \sum_{i=1}^{\infty} e^{-i^{2}}<\infty .
$$

Therefore, (GPC) is satisfied. So $X$ is nuclear, in particular Schwartz.

(ii) $X$ enjoys property (DN): Without loss of any generality assume that $a_{1}(i):=1$. Let us pick $s=1$. For any $i, n \in \mathbb{N}$, and for a constant $C>0$ we clearly have

$$
\begin{aligned}
& \log (2 i n)+i n \leq \log (2 i n)+2 i n \\
& \Rightarrow \quad 2 i n e^{i n} \leq 2 i n e^{2 i n}+\log (C) \\
& \Leftrightarrow \quad e^{2 i n e^{i n}} \leq C e^{2 i n e^{2 i n}} \\
& \Leftrightarrow \quad a_{n}(i)^{2} \leq C a_{2 n}(i) a_{1}(i),
\end{aligned}
$$

With the choice $m=2 n$, we conclude that $X$ has property (DN).

(iii) $X$ is not isomorphic to a power series space of infinite type: Let us define $f: \mathbb{R} \rightarrow \mathbb{R}^{+}$by

$$
f(x):= \begin{cases}x e^{x}, & \text { if } x \geq 0 \\ x e^{-x}, & \text { if } x<0\end{cases}
$$

Clearly $f$ is an odd, positive, increasing, and logarithmically convex function. Then, for $\left(\alpha_{i}\right)_{i \in \mathbb{N}}=(i)_{i \in \mathbb{N}}, X$ is isomorphic to the Dragilev space $L_{f}\left(\alpha_{i}, \infty\right)$ of infinite type. For any $1<\rho<\infty$,

$$
\lim _{x \rightarrow \infty} \frac{f(\rho x)}{f(x)}=\lim _{x \rightarrow \infty} \frac{\rho x e^{\rho x}}{x e^{x}}=\lim _{x \rightarrow \infty} \rho e^{(\rho-1) x}=\infty .
$$

Hence, $f$ is rapidly increasing. By the comments prior to Example 1.3, $X$ cannot be isomorphic to a power series space of infinite type.

\section{Continuity and Compactness of C on $k_{0}(V)$}

An operator $T$ on a Fréchet space $X$ into itself is called bounded (resp. compact) if there exists a neighborhood $U$ of the origin of $X$ such that $T U$ is a bounded (resp. relatively compact) set in $X$. Recall that a Hausdorff inductive limit $E=\operatorname{ind}_{n} E_{n}$ of Banach spaces is called regular if every bounded subset $B$ of $E$ is contained and bounded in some step $E_{n}$. The following lemma is well-known.

Lemma 2.1. Let $E=\operatorname{ind}_{m} E_{m}$ and $F=\operatorname{ind}_{n} F_{n}$ be (LB)-spaces such that $E$ (resp. $F)$ is the union of the sequence of Banach spaces $E_{m}$ (resp. $\left.F_{n}\right)$. Let $T: E \rightarrow F$ be a linear operator. Then

(1) $T$ is continuous if and only if for all $m \in \mathbb{N}$ there exists $n \in \mathbb{N}$ such that $T\left(E_{m}\right) \subset F_{n}$ and $T: E_{m} \rightarrow F_{n}$ is continuous. 
(2) Let $T$ be continuous and let $F$ be regular. Then $T$ is bounded if and only if there exists $n \in \mathbb{N}$ such that for all $m, T\left(E_{m}\right) \subset F_{n}$ and $T: E_{m} \rightarrow F_{n}$ is continuous.

Proposition 2.2. Let $\lambda_{1}(A)$ be a Schwartz Köthe echelon space of order 1. Then, C: $k_{0}(V) \rightarrow k_{0}(V)$ is continuous if and only if for all $n \in \mathbb{N}$ there exists $m>n$ such that

$$
\sup _{i \in \mathbb{N}} \frac{v_{m}(i)}{i} \sum_{j=1}^{i} \frac{1}{v_{n}(j)}<\infty .
$$

Proof. Follows directly from Lemma 2.1, and [3, Proposition 2.2(i)].

Corollary 2.3. Let $A$ be a Köthe matrix satisfying $(G \infty-1)$, and let $\lambda_{1}(A)$ be Schwartz. Then $\mathrm{C} \in \mathcal{L}\left(k_{0}(V)\right)$.

Proof. Since $\lambda_{1}(A)$ is Schwartz, for any $n \in \mathbb{N}$ pick $m>n$ as in condition (S). Hence, $(\mathrm{G} \infty-1)$ yields

$$
\sup _{i \in \mathbb{N}} \frac{v_{m}(i)}{i} \sum_{j=1}^{i} \frac{1}{v_{n}(j)} \leq \sup _{i \in \mathbb{N}} \frac{i v_{m}(i)}{i v_{n}(i)}=\sup _{i \in \mathbb{N}} \frac{v_{m}(i)}{v_{n}(i)}<\infty .
$$

Thus, (2.1) holds, and $\mathrm{C} \in \mathcal{L}\left(k_{0}(V)\right)$ by Proposition 2.2.

Now let us give a characterization for the compactness of $\mathrm{C}$ in $\mathcal{L}\left(k_{0}(V)\right)$. The following proposition is a direct consequence of Lemma 2.1 and [3, Proposition 2.2(ii)], so we omit its proof.

Proposition 2.4. Let $A$ be a Köthe matrix satisfying $(G \infty-1)$, and let $\lambda_{1}(A)$ be Schwartz. Then, C: $k_{0}(V) \rightarrow k_{0}(V)$ is compact if and only if there exists $n \in \mathbb{N}$ such that for all $m>n$ one has

$$
\lim _{i \rightarrow \infty} \frac{v_{m}(i)}{i} \sum_{j=1}^{i} \frac{1}{v_{n}(j)}=0 .
$$

The Köthe echelon space $\lambda_{1}(A)$ of order 1 is said to be a smooth sequence space of finite type (or a $G_{1}$-space) [21, Section 3] if $A$ satisfies

(G1-1) $0<a_{n}(i+1) \leq a_{n}(i)$, for all $n \in \mathbb{N}$ and $i \in \mathbb{N}$.

(G1-2) For all $n \in \mathbb{N}$ there exist $m>n$ and $C>0$ such that $a_{n}(i) \leq C a_{m}(i)^{2}$, for all $i \in \mathbb{N}$.

The Cesàro operator on $G_{1}$-spaces was studied by the author in [15]. The following proposition shows that $\mathrm{C}$ is not continuous on duals of nuclear $G_{1}$-spaces.

Proposition 2.5. Let $A$ be a Köthe matrix satisfying (G1-1), and suppose $\lambda_{1}(A)$ is nuclear. Then, the Cesàro operator $\mathrm{C}$ does not belong to $\mathcal{L}\left(k_{0}(V)\right)$.

Proof. Since $k_{0}(V)$ is nuclear, by [15, Theorem 1], $\frac{i}{v_{n}(i)} \stackrel{i}{\rightarrow} 0$, for all $n \in \mathbb{N}$. Now suppose $\mathrm{C}$ is continuous on $k_{0}(V)$. Then by Proposition 2.2 and (G1-1), for $n=1$, there exists $m>1$ such that

$$
M v_{1}(1) \geq v_{1}(1) \frac{v_{m}(i)}{i} \sum_{j=1}^{i} \frac{1}{v_{1}(j)} \geq \frac{v_{m}(i)}{i v_{1}(1)} v_{1}(1) \stackrel{i}{\rightarrow} \infty,
$$

for a constant $M>0$. This is a contradiction. Hence, $\mathrm{C} \notin \mathcal{L}\left(k_{0}(V)\right)$. 
Let $D: \mathbb{C}^{\mathbb{N}} \rightarrow \mathbb{C}^{\mathbb{N}}$ be the formal operator of differentiation defined by $D\left(x_{1}, x_{2}, x_{3}, \ldots\right)=$ $\left(x_{2}, 2 x_{3}, 3 x_{4}, \ldots\right), x=\left(x_{i}\right)_{i} . D$ is closely related to the Cesàro operator $\mathrm{C} \in \mathcal{L}\left(\mathbb{C}^{\mathbb{N}}\right)$ by the identity $\mathrm{C}^{-1}=\left(I-S_{r}\right) \circ D \circ S_{r}$, where $S_{r} \in \mathcal{L}\left(\mathbb{C}^{\mathbb{N}}\right)$ is the right-shift operator. The following result is proved via a similar argument in [15, Proposition 7].

Proposition 2.6. Let $A$ be a Köthe matrix. Then, for the co-echelon space $k_{0}(V)$ and the formal differentiation operator $D$, the following statements are equivalent:

(1) The differentiation operator $D: k_{0}(V) \rightarrow k_{0}(V)$ is continuous.

(2) For all $n \in \mathbb{N}$ there exist $m>n$ and $M>0$ such that

$$
\sup _{i \in \mathbb{N}} i v_{m}(i) \leq M v_{n}(i+1) .
$$

Example 2.7. Consider the nuclear $G_{\infty}$-space $X$ constructed in Example 1.3. For $Y:=k_{0}(V)=\left(\lambda_{1}(A)\right)^{\prime}=X^{\prime}$, choose $m=2 n$ to observe

$$
\begin{aligned}
\frac{i v_{m}(i)}{v_{n}(i+1)}=\frac{i \exp \left(-2 i n e^{2 i n}\right)}{\exp \left(-i n e^{(i+1) n}\right)} & =\exp \left(\log (i)+i n e^{(i+1) n}-2 i n e^{2 i n}\right) \\
& =\exp \left(\left(\frac{\log (i)}{i n e^{2 i n}}+e^{n} e^{-i n}-2\right) i n e^{2 i n}\right) \stackrel{i}{\rightarrow} 0 .
\end{aligned}
$$

Hence, $D: Y \rightarrow Y$ is continuous.

Proposition 2.8. For a $G_{\infty}$-Köthe matrix $A$, and the associated co-echelon space $k_{0}(V)$, the following statements are equivalent:

(1) $k_{0}(V)$ is nuclear.

(2) For all $n \in \mathbb{N}$ there exists $m>n$ such that

$$
\sup _{i \in \mathbb{N}} \frac{i v_{m}(i)}{v_{n}(i)}<\infty .
$$

(3) Given $\alpha \in \mathbb{R}$, for all $n \in \mathbb{N}$ there exists $m>n$ such that

$$
\sup _{i \in \mathbb{N}} \frac{i^{\alpha} v_{m}(i)}{v_{n}(i)}<\infty .
$$

Proof. The proof reads as [15, Proposition 9]. In the implication (1) $\Rightarrow(2)$ if we set $b_{n}(i):=\prod_{j=1}^{n} a_{j}(i)$, the rest follows with the same arguments.

Proposition 2.9. Given a real number $\alpha \geq 1$. Then, for a $G_{\infty}$-Köthe matrix $A$, and the associated co-echelon space $k_{0}(V)$, the following statements are equivalent:

(1) $k_{0}(V)$ is nuclear.

(2) There exists $n \in \mathbb{N}$ such that $\lim _{i \rightarrow \infty} i^{\alpha} v_{n}(i)=0$.

(3) There exists $n \in \mathbb{N}$ such that $\lim _{i \rightarrow \infty} i^{\alpha} v_{n}(i)=L<\infty$.

Proof. (1) $\Rightarrow(2)$ Let $k_{0}(V)$ be nuclear. For any $n \in \mathbb{N}$ pick $m>n$ as in $(\mathrm{N})$. Then, by $(\mathrm{G} \infty-1)$ and proof of $[15$, Proposition 9$]$, we have

$$
i^{\alpha} v_{m}(i) \leq \frac{i^{\alpha} v_{m}(i)}{v_{n}(i)} \stackrel{i}{\rightarrow} 0 .
$$

(2) $\Rightarrow(3)$ Trivial.

(3) $\Rightarrow(1)$ Let us have $n \in \mathbb{N}$ with $i^{\alpha} v_{n}(i) \stackrel{i}{\rightarrow} L$, for a given $\alpha>1$. For this $n$ we pick $m>n$ and $C>0$ as in $(\mathrm{G} \infty-2)$ and similarly we find $p>m$ and $M>0$ as in 
$(\mathrm{G} \infty-2)$. Then, for all $i \in \mathbb{N}$,

$$
\frac{v_{p}(i)}{v_{n}(i)} \leq C \frac{v_{p}(i)}{v_{m}(i)} \leq C M \frac{v_{m}(i)^{2}}{v_{m}(i)} \leq C M v_{n}(i) \leq \frac{C M L}{i^{\alpha}} \in \ell_{1} .
$$

Hence, $\left(\frac{v_{p}(i)}{v_{n}(i)}\right)_{i} \in \ell_{1}$ and so (GPC) is satisfied. Therefore, $\lambda_{1}(A)$ is nuclear.

\section{Spectrum of C in the nuclear Case}

For a locally convex Hausdorff space $X$ and $T \in \mathcal{L}(X)$, the resolvent set $\rho(X ; T)$ of $T$ consists of all $\lambda \in \mathbb{C}$ such that $(\lambda I-T)^{-1}$ exists in $\mathcal{L}(X)$. The set $\sigma(T ; X):=$ $\mathbb{C} \backslash \rho(T ; X)$ is called the spectrum of $T$ on $X$. The point spectrum $\sigma_{p t}(T ; X)$ of $T$ on $X$ consists of all $\lambda \in \mathbb{C}$ such that $(\lambda I-T)$ is not injective. Unlike for Banach spaces, it might happen that $\rho(T ; X)=\varnothing$ or that $\rho(T ; X)$ is not open in $\mathbb{C}$. That is why some authors prefer the subset $\rho^{*}(T ; X)$ of $\rho(T ; X)$ consisting of all $\lambda \in \mathbb{C}$ for which there exists $\delta>0$ such that the open disk $B(\lambda, \delta):=\{z \in \mathbb{C}:|z-\lambda|<$ $\delta\} \subseteq \rho(T)$ and $\{R(\mu, T): \mu \in B(\lambda, \delta)\}$ is equicontinuous in $\mathcal{L}(X)$. We denote $\Sigma:=\left\{\frac{1}{m}: m \in \mathbb{N}\right\}$ and $\Sigma_{0}:=\Sigma \cup\{0\}$. In this section we investigate the spectra $\sigma_{p t}\left(\mathrm{C} ; k_{0}(V)\right), \sigma^{*}\left(\mathrm{C} ; k_{0}(V)\right)$, and $\sigma\left(\mathrm{C} ; k_{0}(V)\right)$ in case $k_{0}(V)$ is nuclear (equivalently, $\lambda_{1}(A)$ is nuclear).

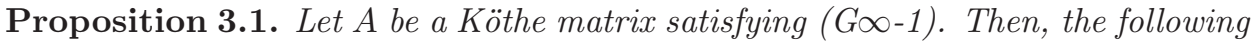
statements are equivalent:

(1) $0 \notin \sigma\left(\mathrm{C} ; k_{0}(V)\right)$.

(2) A satisfies condition $(\mathrm{N})$.

Proof. $0 \notin \sigma\left(\mathrm{C} ; k_{0}(V)\right)$ if and only if $\mathrm{C}^{-1}: k_{0}(V) \rightarrow k_{0}(V)$ is continuous if and only if for all $n \in \mathbb{N}$ there exists $m>n$ such that $\mathrm{C}^{-1}: c_{0}\left(v_{n}\right) \rightarrow c_{0}\left(v_{m}\right)$ is continuous. Hence the proof proceeds as in [15, Proposition 10].

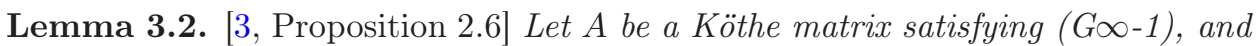
let $\lambda_{1}(A)$ be Schwartz. Then for $s \in \mathbb{N}$ and for the Cesàro operator $\mathrm{C}$, the following statements are equivalent:

(1) $\frac{1}{s+1} \in \sigma_{p t}\left(\mathrm{C} ; k_{0}(V)\right)$.

(2) There exists $n \in \mathbb{N}$ such that $\lim _{i \rightarrow \infty} i^{s} v_{n}(i)=0$.

Proposition 3.3. Let $A$ be a $(G \infty-1)$ Köthe matrix satisfying $(\mathrm{N})$, and let $\lambda_{1}(A)$ be Schwartz. Then, $\Sigma=\sigma_{p t}\left(\mathrm{C} ; k_{0}(V)\right)$.

Proof. We clearly have $\sigma_{p t}\left(\mathrm{C} ; k_{0}(V)\right) \subseteq \sigma_{p t}\left(\mathrm{C}, \mathbb{C}^{\mathbb{N}}\right)=\Sigma$. Now we prove that there exists $n \in \mathbb{N}$ such that for all $s \in \mathbb{N}$ we have $i^{s} v_{n}(i) \stackrel{i}{\rightarrow} 0$, by induction over $s$. Since $\lambda_{1}(A)$ is Schwartz, we may choose $n \in \mathbb{N}$ as in condition (I) so that $v_{n}(i) \stackrel{i}{\rightarrow} 0$. Then, by assumption there exist $m_{1}>n$ and $M>0$ such that $i v_{m_{1}}(i) \leq M v_{n}(i)$ and hence $i v_{m_{1}}(i) \stackrel{i}{\rightarrow} 0$. Suppose that $i^{s} v_{m_{1}}(i) \stackrel{i}{\rightarrow} 0$, for $s=1, \ldots, r$. Then, there exist $m_{2}>m_{1}$ and $\tilde{M}$ satisfying

$$
i^{r+1} v_{m_{2}}(i)=i^{r} i v_{m_{2}}(i) \leq \tilde{M} i^{r} v_{m_{1}}(i) \stackrel{i}{\rightarrow} 0 .
$$

That implies for some $n \in \mathbb{N}$, we have $i^{s} v_{n}(i) \stackrel{i}{\rightarrow} 0$, for all $s \in \mathbb{N}$. Equivalently, by Lemma 3.2, $\frac{1}{s+1} \in \sigma_{p t}\left(\mathbf{C} ; k_{0}(V)\right)$, for all $s \in \mathbb{N}$. Therefore $\Sigma=\sigma_{p t}\left(\mathbf{C} ; k_{0}(V)\right)$.

Theorem 3.4. Let $\lambda_{1}(A)$ be a $G_{\infty}$-space which is Schwartz. Then, the following statements are equivalent: 
(1) $0 \notin \sigma\left(\mathrm{C} ; k_{0}(V)\right)$.

(2) $\frac{1}{2} \in \sigma_{p t}\left(\mathrm{C} ; k_{0}(V)\right)$.

(3) There exists $s \in \mathbb{N}$ such that $\frac{1}{s} \in \sigma_{p t}\left(\mathrm{C} ; k_{0}(V)\right)$.

(4) $\Sigma=\sigma_{p t}\left(\mathrm{C} ; k_{0}(V)\right)$.

Proof. (1) $\Rightarrow(2)$ Proposition 2.8 and Proposition 3.1 yield $k_{0}(V)$ is nuclear. Then, by Proposition 1.2 we may take $n \in \mathbb{N}$ as in $(\mathrm{SV})$, so that $\left(v_{n}(i)\right)_{i} \in \ell_{1}$. We may also pick $m>n$ and $M>0$ as in (N) so that $i v_{m}(i) \leq M v_{n}(i)$, for all $i \in \mathbb{N}$. Then, we have $\left(i v_{m}(i)\right)_{i} \in \ell_{1}$. This implies $i v_{m}(i) \stackrel{i}{\rightarrow} 0$. This is equivalent to (2) by Lemma 3.2 .

$(2) \Rightarrow(1)$ Since $\frac{1}{2} \in \sigma_{p t}\left(\mathrm{C} ; k_{0}(V)\right)$, by Lemma 3.2 there is an $n \in \mathbb{N}$ such that $i v_{n}(i) \stackrel{i}{\rightarrow} 0$. If we select $m>n$ and $M>0$ as in $(\mathrm{G} \infty-2)$, we obtain

$$
\frac{i v_{m}(i)}{v_{n}(i)} \leq M i v_{n}(i) \stackrel{i}{\rightarrow} 0 .
$$

This is equivalent to (1) by Proposition 3.1.

$(2) \Rightarrow(3)$ Clear.

(3) $\Rightarrow(4)$ By Lemma 3.2 we have an $s \in \mathbb{N}$ with $i^{s} v_{n}(i) \stackrel{i}{\rightarrow} 0$ for some $n \in \mathbb{N}$. Then, clearly $i v_{n}(i) \stackrel{i}{\rightarrow} 0$ as well. Now let us prove by induction that $i^{2^{k}} v_{n}(i) \stackrel{i}{\rightarrow} 0$ for all $k \in \mathbb{N}$. For $k=0$, it is already satisfied. Suppose $i^{2^{k}} v_{n}(i) \stackrel{i}{\rightarrow} 0$, for $k=1, \ldots, r$. Then, for some $i_{0} \in \mathbb{N}$, we have $\left|i^{2^{r}} v_{n}(i)\right|<1$ for all $i \geq i_{0}$. For $m>n$ and $C>0$ selected as in $(\mathrm{G} \infty-2)$ and for all $i \geq i_{0}$,

$$
i^{2^{r+1}} v_{m}(i)=\left(i^{2^{r}} \sqrt{v_{m}(i)}\right)^{2} \leq C\left(i^{2^{r}} v_{n}(i)\right)^{2} \leq C i^{2^{r}} v_{n}(i) \stackrel{i}{\rightarrow} 0 .
$$

Therefore $\frac{1}{s} \in \sigma_{p t}\left(\mathrm{C} ; k_{0}(V)\right)$ for all $s \in \mathbb{N}$. Hence, $\sigma_{p t}\left(\mathrm{C} ; k_{0}(V)\right)=\Sigma$.

$(4) \Rightarrow(2)$ Trivial.

The following example illustrates why assumption $(\mathrm{G} \infty-2)$ in Theorem 3.4 cannot be removed.

Example 3.5. (i) For a fixed $0<\alpha<1$, and an increasing sequence $\left(\alpha_{n}\right)_{n} \subset(0,1)$ tending to $\alpha$, let us define a Köthe matrix $A$ by $a_{n}(i):=i^{\alpha_{n}} e^{i}$, where $i, n \in \mathbb{N}$. The Köthe echelon space $\lambda_{1}(A)$ of order 1 satisfies condition $(\mathrm{G} \infty-1)$ and condition (I). Assume that $(\mathrm{G} \infty-2)$ also holds. Then, given $n=1$ there is $m>1$ and $M>0$ with

$$
i^{2 \alpha_{1}} e^{2 i} \leq M i^{\alpha_{n}} e^{i} \Rightarrow i^{\alpha_{1}} e^{i} \leq M i^{\alpha_{n}-\alpha_{1}},
$$

which is impossible. Hence $A$ is not a $G_{\infty}$-matrix. For $n=1$,

$$
\sup _{i \in \mathbb{N}} \frac{i^{1+\alpha_{1}} e^{i}}{i^{\alpha_{m}} e^{i}}=\sup _{i \in \mathbb{N}} i^{1+\alpha_{1}-\alpha_{m}}=\infty, \quad \forall m>1,
$$

since $1+\alpha_{1}-\alpha_{m}>0$, for all $m>1$. So (N) is not satisfied. On the other hand, for each $s, n \in \mathbb{N}$ and for large values of $i \in \mathbb{N}$, we have $0<i^{s-1} v_{n}(i)=i^{s-1-\alpha_{n}} e^{-i} \leq$ $i^{s-1} e^{-i} \stackrel{i}{\rightarrow} 0$. So $\left(i^{s-1}\right)_{i} \in k_{0}(V)$ and by Lemma $3.2 \frac{1}{s} \in \sigma_{p t}\left(\mathrm{C} ; k_{0}(V)\right)$ for each $s \in \mathbb{N}$. This shows that condition (4) does not imply condition (1) in Theorem 3.4, in general.

(ii) Fix $s \geq 1, s \in \mathbb{N}$ and define the Köthe matrix $A=\left(a_{n}\right)_{n}$ by $a_{n}(i):=i^{s-\frac{1}{1+n}}$. The Köthe echelon space $\lambda_{1}(A)$ of order 1 satisfies $(\mathrm{G} \infty-1)$ and condition (I), but 
it is not a $G_{\infty}$-space. Indeed, assume that $(\mathrm{G} \infty-2)$ holds. Then for $n=1$, there exist $m>1$ and $M>0$ such that $a_{1}(i)^{2} \leq M a_{m}(i)$. So for any $s \geq 1$,

$$
i^{2 s-1} \leq M i^{s-\frac{1}{1+m}} \quad \Rightarrow \quad i^{s} \leq M i^{1-\frac{1}{1+m}} .
$$

But this is impossible since $s \geq 1$. In this case, $\left(i^{m-1}\right)_{i} \in k_{0}(V)$ for $m=1,2, \ldots s$ but $\left(i^{s}\right)_{i} \notin k_{0}(V)$ since $i^{s} v_{n}(i)=i^{\frac{1}{1+n}} \stackrel{i}{\rightarrow} \infty$, for all $n \in \mathbb{N}$. Thus $\frac{1}{s+1} \notin$ $\sigma_{p t}\left(\mathrm{C} ; k_{0}(V)\right)$, which implies $\frac{1}{m} \notin \sigma_{p t}\left(\mathrm{C} ; k_{0}(V)\right)$ for each $m>s$. This shows us condition (3) in Theorem 3.4 does not imply (4), in general.

Let us define the continuous function $a: \mathbb{C} \backslash\{0\} \rightarrow \mathbb{R}$ by

$$
a(z):=\operatorname{Re}\left(\frac{1}{z}\right) .
$$

Observe that for all $k \in \mathbb{N}$, the weighted Banach space $c_{0}\left(v_{k}\right)$ is isometrically isomorphic to $c_{0}$ via $\phi_{k}: c_{0}\left(v_{k}\right) \rightarrow c_{0}$ defined by

$$
\phi_{k} x:=\left(v_{k}(i) x_{i}\right)_{i}, \quad \forall x \in c_{0}\left(v_{k}\right) .
$$

Proposition 3.6. Let $\lambda_{1}(A)$ be a nuclear $G_{\infty}$-space. Then,

(1) $\sigma\left(\mathrm{C} ; k_{0}(V)\right)=\sigma_{p t}\left(\mathrm{C} ; k_{0}(V)\right)=\Sigma$.

(2) $\sigma^{*}\left(\mathrm{C} ; k_{0}(V)\right)=\sigma\left(\mathrm{C} ; k_{0}(V)\right) \cup\{0\}=\Sigma_{0}$.

Proof. Since $k_{0}(V)$ is nuclear, by Theorem 3.4, we know that $\sigma_{p t}\left(\mathrm{C} ; k_{0}(V)\right)=\Sigma \subseteq$ $\sigma\left(\mathrm{C} ; k_{0}(V)\right)$, and hence

$$
\Sigma_{0}=\bar{\Sigma} \subseteq \overline{\sigma\left(\mathrm{C} ; k_{0}(V)\right)} \subseteq \sigma^{*}\left(\mathrm{C} ; k_{0}(V)\right) .
$$

Moreover, Proposition 3.1 yields $0 \notin \sigma\left(\mathrm{C} ; k_{0}(V)\right)$. For the other inclusion, we show that for every $\lambda \in \mathbb{C} \backslash \Sigma_{0}$ there exists $\delta>0$ such that the inverse operator $(\mathrm{C}-\mu I)^{-1}: k_{0}(V) \rightarrow k_{0}(V)$ is continuous for each $\mu \in B(\lambda, \delta)$ and the set $\{(\mathrm{C}-$ $\left.\mu I)^{-1}: \mu \in B(\lambda, \delta)\right\}$ is equicontinuous in $\mathcal{L}\left(k_{0}(V)\right)$. Remember that $(\mathrm{C}-\mu I)^{-1}$ is continuous on $\mathbb{C}^{\mathbb{N}}$ for each $\mu \in \mathbb{C} \backslash \Sigma$. Fix $\lambda \in \mathbb{C} \backslash \Sigma_{0}$. Choose a $\delta_{1}>0$ such that $B(\lambda, \delta) \cap \Sigma_{0}=\varnothing$. To establish our claim, it suffices to show that there exists $\delta>0$ such that for all $n \in \mathbb{N}$ there exist $m>n$ and $D_{n}>0$ satisfying

$$
q_{m}\left((\mathrm{C}-\mu I)^{-1} x\right) \leq D_{n} q_{n}(x), \quad \forall \mu \in B(\lambda, \delta), x \in c_{0}\left(v_{n}\right) .
$$

Now we separate in two cases.

(i) $a(\lambda)<1$ (equivalently, $\left|\lambda-\frac{1}{2}\right|>\frac{1}{2}$ ): Fix $n \in \mathbb{N}$. Since $a(\lambda)<1$ we may pick $\varepsilon>0$ such that $a(\lambda)<1-\varepsilon$. By continuity of $a$, there exists $\delta_{2}>0$ such that $a(\mu)<1-\varepsilon$, for all $\mu \in B\left(\lambda, \delta_{2}\right)$. By [5, Lemma 2.8], there exist $\delta \in\left(0, \delta_{2}\right)$ and $M_{n, \lambda}$ such that (3.3) is satisfied:

$$
q_{n}\left((\mathrm{C}-\mu I)^{-1} x\right) \leq \frac{M_{n, \lambda}}{1-a(\mu)} q_{n}(x) \leq \frac{M_{n, \lambda}}{\varepsilon} q_{n}(x), \quad \forall \mu \in \overline{B(\lambda, \delta)}, x \in c_{0}\left(v_{n}\right) .
$$

(ii) $a(\lambda) \geq 1$ (equivalently, $\left|\lambda-\frac{1}{2}\right| \leq \frac{1}{2}$ ): Let us recall the formula for the operator $(\mathrm{C}-\mu I)^{-1}: \mathbb{C}^{\mathbb{N}} \rightarrow \mathbb{C}^{\mathbb{N}}$ whenever $\mu \notin \Sigma_{0}$. By [19], the $i$-th row of the matrix for 
$(\mathrm{C}-\mu I)^{-1}$ has the entries:

$$
\begin{cases}\frac{-1}{i \mu^{2} \prod_{k=j}^{i}\left(1-\frac{1}{k \mu}\right)} & \text { if } 1 \leq j<i \\ \frac{1}{\frac{1}{i}-\mu} & \text { if } i=j \\ 0 & \text { otherwise. }\end{cases}
$$

For $D_{\mu}=\left(d_{i j}\right)_{i, j}$ and $E_{\mu}=\left(e_{i j}\right)_{i, j}$, one may formulate $(\mathrm{C}-\mu I)^{-1}=D_{\mu}-\frac{1}{\mu^{2}} E_{\mu}$, where $d_{i j}=\frac{1}{\frac{1}{i}-\mu}$, for $i=j$ otherwise $d_{i j}=0$; and $e_{i j}=\frac{1}{i \prod_{k=j}\left(1-\frac{1}{k \mu}\right)}$, for $2 \leq j<i$ otherwise $e_{i j}=0$. Define $d_{0}(\lambda):=\operatorname{dist}\left(B(\lambda, \delta), \Sigma_{0}\right)>0$. We have $\left|d_{i i}\right|<\frac{1}{d_{0}(\lambda)}$, for all $\mu \in \overline{B\left(\lambda, \delta_{1}\right)}$ and $i \in \mathbb{N}$. Fix $n \in \mathbb{N}$. Then for every $x \in c_{0}\left(v_{n}\right)$ and $\mu \in \overline{B\left(\lambda, \delta_{1}\right)}$

$$
q_{n}\left(D_{\mu}(x)\right)=\sum_{i=1}^{\infty}\left|d_{i i}(\mu) x_{i}\right| v_{n}(i) \leq \frac{1}{d_{0}(\lambda)} \sum_{i=1}^{\infty}\left|x_{i}\right| v_{n}(i)=\frac{1}{d_{0}(\lambda)} q_{n}(x)
$$

So $\left\{D_{\mu}: \mu \in \overline{B\left(\lambda, \delta_{1}\right)}\right\} \subseteq \mathcal{L}\left(c_{0}\left(v_{m}\right)\right)$. Then, it remains to show that $E_{\mu}: k_{0}(V) \rightarrow$ $k_{0}(V)$ is continuous for all $\mu \in \overline{B(\lambda, \delta)}$ for some $\delta>0$. So by (3.2), it suffices to show that for all $n \in \mathbb{N}$ there exist $m \geq n$ and $D_{n}>0$ such that

$$
\left\|\phi_{m} \circ E_{\mu} \circ \phi_{n}^{-1} x\right\|_{0} \leq D_{n}\|x\|_{0}, \quad \forall x \in c_{0}, \mu \in \overline{B\left(\lambda, \delta_{1}\right)} .
$$

where $\|\cdot\|_{0}$ is the usual $c_{0}$-norm. For each $n, m$ define $\tilde{E}_{\mu, n, m}:=\phi_{m} \circ E_{\mu} \circ \phi_{n}^{-1} \in$ $\mathcal{L}\left(\mathbb{C}^{\mathbb{N}}\right)$ for $\mu \in \mathbb{C}^{\mathbb{N}} \backslash\{0\}$. Fix $n \in \mathbb{N}$. For each $m \geq n$ the operator $\tilde{E}_{\mu, m, n}$ for $\mu \in B\left(\lambda, \delta_{1}\right)$ is the restriction to $c_{0}$ of

$$
\tilde{E}_{\mu, m, n}(x)=\left(\tilde{E}_{\mu, m, n}(x)\right)=\left(v_{m}(i) \sum_{j=1}^{i-1} \frac{e_{i j}(\mu)}{v_{n}(j)} x_{j}\right), \quad x \in \mathbb{C}^{\mathbb{N}},
$$

with $\left(\tilde{E}_{\mu, m, n}\right)_{1}:=0 . \quad \tilde{E}_{\mu, m, n}=\left(\tilde{e}_{i j}^{n m}(\mu)\right)$ is given by $\tilde{e}_{1 j}^{n m}=0, \tilde{e}_{i j}^{n m}=\frac{v_{m}(i)}{v_{n}(j)} e_{i j}(\mu)$ for $i \geq 2$ and $1 \leq j<i$. So it suffices to verify, for some $m \geq n$ and $\delta>0$ one has $\tilde{E}_{\mu, m, n} \in \mathcal{L}\left(c_{0}\right)$ for $\mu \in B(\lambda, \delta)$, and $\left\{\tilde{E}_{\mu, m, n}: \mu \in B(\lambda, \delta)\right\}$ is equicontinuous in $\mathcal{L}\left(c_{0}\right)$. To prove this, we observe [5, Lemma 2.7] implies that for every $m \geq n$, and all $\mu \in \overline{B\left(\lambda, \delta_{2}\right)}$ that

$$
\left|\tilde{e}_{i j}^{n m}(\mu)\right|=\frac{v_{m}(i)}{v_{n}(j)}\left|e_{i j}(\mu)\right| \leq D_{\lambda}^{\prime} \frac{i^{a(\mu)-1} v_{m}(i)}{j^{a(\mu)} v_{n}(j)},
$$

for some $D_{\lambda}^{\prime}>0$ and $\delta_{2} \in\left(0, \delta_{1}\right)$. Since $a$ is continuous, there exists $\delta \in\left(0, \delta_{2}\right)$ such that $a(\lambda)-\frac{1}{2}<a(\mu)<a(\lambda)+\frac{1}{2}$ for all $\mu \in \overline{B(\lambda, \delta)}$. Then $a(\mu)>a(\lambda)-\frac{1}{2} \geq \frac{1}{2}$. By picking $m>n$ and $M>0$ as in (SN), for any $\mu \in \overline{B(\lambda, \delta)}$ we have

$$
\left|\tilde{e}_{i j}^{n m}(\mu)\right| \leq D_{\lambda}^{\prime} \frac{v_{m}(i)}{v_{n}(j)} \frac{i^{a(\mu)-1}}{j^{a(\mu)}} \leq \frac{D_{\lambda}^{\prime}}{j^{a(\mu)}} \frac{i^{a(\mu)} v_{m}(i)}{i v_{n}(i)} \leq \frac{M D_{\lambda}^{\prime}}{i} \stackrel{i}{\rightarrow} 0,
$$


Moreover, employing (3.5) and (Gळ-1), respectively, we obtain

$$
\begin{aligned}
\sup _{i \in \mathbb{N}} \sum_{j=1}^{\infty}\left|\tilde{e}_{i j}^{n m}(\mu)\right| & \leq \sup _{i \in \mathbb{N}} D_{\lambda}^{\prime} i^{a(\mu)-1} \sum_{j=1}^{i-1} \frac{v_{m}(i)}{j^{a(\mu)} v_{n}(j)} \leq \sup _{i \in \mathbb{N}} D_{\lambda}^{\prime} i^{a(\mu)-1} v_{m}(i) \frac{i-1}{v_{n}(i)} \\
& \leq \sup _{i \in \mathbb{N}} D_{\lambda}^{\prime} \frac{i^{a(\mu)} v_{m}(i)}{v_{n}(i)}<\infty,
\end{aligned}
$$

for every $\mu \in \overline{B(\lambda, \delta)}$. Hence, [3, Lemma 2.1] implies that satisfying both (3.6) and (3.7) yields $\tilde{E}_{\mu, m, n} \in \mathcal{L}\left(c_{0}\right)$ for all $\mu \in \overline{B(\lambda, \delta)}$. Moreover, the operator norm is given by $\left\|\tilde{E}_{\mu, m, n}\right\|=\sup _{i \in \mathbb{N}} \sum_{j=1}^{i}\left|\tilde{e}_{i j}^{n m}(\mu)\right|$, and we have shown that there exists $C>0$ such that $\left\|\tilde{E}_{\mu, m, n}\right\| \leq C D_{\lambda}^{\prime}$, for all $\mu \in \overline{B(\lambda, \delta)}$. This implies $\left\{\tilde{E}_{\mu, m, n}: \mu \in \overline{B(\lambda, \delta)}\right\}$ is equicontinuous in $\mathcal{L}\left(c_{0}\right)$.

Corollary 3.7. Let $\lambda_{1}(A)$ be a nuclear $G_{\infty}$-space. Then $\mathrm{C} \in \mathcal{L}\left(k_{0}(V)\right)$ is neither compact nor weakly compact.

Proof. Since $k_{0}(V)$ is a Montel space, there is no distinction between compactness and weak compactness. So, suppose $\mathrm{C}$ is compact. Then $\sigma\left(\mathrm{C} ; k_{0}(V)\right)$ is necessarily a compact set in $\mathbb{C}[10$, Theorem 9.10.2]. This contradicts Proposition 3.6.

When acting on $\mathbb{C}^{\mathbb{N}}$, the Cesàro matrix $\mathrm{C}$ is similar to the diagonal matrix $\operatorname{diag}\left(\frac{1}{i}\right)$. Indeed, the identity $\mathrm{C}=\Delta \operatorname{diag}\left(\frac{1}{i}\right) \Delta$ holds in $\mathcal{L}\left(\mathbb{C}^{\mathbb{N}}\right)$, where

$$
\Delta=\Delta^{-1}=\left(\Delta_{i j}\right)_{i, j \in \mathbb{N}}= \begin{cases}(-1)^{j-1}\left(\begin{array}{l}
i-1 \\
j-1
\end{array}\right), & \text { if } 1 \leq j<i \\
0, & \text { if } j>i\end{cases}
$$

and all the three operators $C, \operatorname{diag}\left(\frac{1}{i}\right)$, and $\Delta$ are continuous.

Proposition 3.8. For a $G_{\infty}$-Köthe matrix $A$, and for the operator $\Delta$, the following statements are equivalent:

(1) There exists $n \in \mathbb{N}$ such that

$$
\sup _{i \in \mathbb{N}} i^{i} v_{n}(i)<\infty
$$

(2) $\Delta \in \mathcal{L}\left(k_{0}(V)\right)$.

Proof. For every $k \in \mathbb{N}$, the surjective isomorphism $\phi_{k}: c_{0}\left(v_{k}\right) \rightarrow c_{0}$ is defined by (3.2). Since $k_{0}(V)=\operatorname{ind}_{n} c_{0}\left(v_{n}\right)$, we have $\Delta \in \mathcal{L}\left(k_{0}(V)\right)$ if and only if for all $n \in \mathbb{N}$ there exists $m>n$ with $\Delta: c_{0}\left(v_{n}\right) \rightarrow c_{0}\left(v_{m}\right)$ is continuous if and only if the operator $D^{n m}: c_{0} \rightarrow c_{0}$ defined by $D^{n m}:=\phi_{m} \circ \Delta \circ \phi_{n}^{-1}$ is continuous, where $\phi_{m}=\operatorname{diag}\left(v_{m}(i)\right)$ and $\phi_{n}^{-1}=\operatorname{diag}\left(\frac{1}{v_{n}(i)}\right)$. Hence, $D^{n m}$ has a lower triangular matrix whose entries are given by

$$
d_{i j}^{n m}=(-1)^{j-1} \frac{v_{m}(i)}{v_{n}(j)}\left(\begin{array}{c}
i-1 \\
j-1
\end{array}\right), \quad 1 \leq j<i
$$

and $d_{i j}^{n m}=0$ for $j \geq i$. It follows by [20, Theorem 4.51-C] that $\Delta \in \mathcal{L}\left(k_{0}(V)\right)$ if and only if for each $n \in \mathbb{N}$ we find $m>n$ so that both (3.8) and (3.9) hold:

$$
\lim _{i \rightarrow \infty}\left|d_{i j}^{n m}\right|=\lim _{i \rightarrow \infty} \frac{v_{m}(i)}{v_{n}(j)}\left(\begin{array}{c}
i-1 \\
j-1
\end{array}\right)=0, \quad \forall j \in \mathbb{N},
$$




$$
\sup _{i \in \mathbb{N}} \sum_{j=1}^{\infty}\left|d_{i j}^{n m}\right|=\sup _{i \in \mathbb{N}} \sum_{j=1}^{i} \frac{v_{m}(i)}{v_{n}(j)}\left(\begin{array}{l}
i-1 \\
j-1
\end{array}\right)<\infty
$$

Observe that

$$
\begin{aligned}
\left(\begin{array}{l}
i-1 \\
j-1
\end{array}\right) & =\frac{(i-1) !}{(j-1) !(i-j) !}=\frac{(i-1) \cdots(i-j+1)}{(j-1) !} \\
& =\frac{i^{j-1}}{(j-1) !}\left(1-\frac{1}{i}\right) \cdots\left(1-\frac{j-1}{i}\right) .
\end{aligned}
$$

$(1) \Rightarrow(2)$ Let us assume that there exists $n_{0} \in \mathbb{N}$ as in condition (U). Then, $\sup _{i \in \mathbb{N}} i^{i} v_{n}(i)<\infty$, for every $n \geq n_{0}$. In particular, $\lim _{i \rightarrow \infty} i^{\alpha} v_{n}(i)=0$, for a given real number $\alpha>1$. First using $(\mathrm{G} \infty-1)$ then (3.10) and then given $n \geq n_{0}$, taking $m>n$ and $C>0$ as in $(\mathrm{G} \infty-2)$ yield

$$
\frac{v_{m}(i)}{v_{n}(j)}\left(\begin{array}{l}
i-1 \\
j-1
\end{array}\right) \leq \frac{v_{m}(i)}{v_{n}(i)}\left(\begin{array}{l}
i-1 \\
j-1
\end{array}\right) \leq C v_{n}(i) \frac{i^{j-1}}{(j-1) !}\left(1-\frac{1}{i}\right) \cdots\left(1-\frac{j-1}{i}\right) \stackrel{i}{\rightarrow} 0,
$$

for all $j \in \mathbb{N}$. This shows that (3.8) is satisfied. To prove that (3.9) also holds, we first use $(\mathrm{G} \infty-1)$, then given $n \geq n_{0}$ we choose $m>n$ and $\tilde{C}>0$ as in $(\mathrm{G} \infty-2)$, and then apply (3.10) to get

$$
\begin{aligned}
\sup _{i \in \mathbb{N}} \sum_{j=1}^{i} \frac{v_{m}(i)}{v_{n}(j)}\left(\begin{array}{l}
i-1 \\
j-1
\end{array}\right) & \leq \sup _{i \in \mathbb{N}} \frac{v_{m}(i)}{v_{n}(i)} \sum_{j=1}^{i}\left(\begin{array}{c}
i-1 \\
j-1
\end{array}\right) \\
& \leq \tilde{C} \sup _{i \in \mathbb{N}} v_{n}(i) \sum_{j=1}^{i}\left[\frac{i^{j-1}}{(j-1) !}\left(1-\frac{1}{i}\right) \cdots\left(1-\frac{j-1}{i}\right)\right] \\
& \leq \tilde{C} \sup _{i \in \mathbb{N}} i^{i} v_{n}(i)\left(1-\frac{1}{i}\right)<\infty,
\end{aligned}
$$

by (1). Therefore, $\Delta \in \mathcal{L}\left(k_{0}(V)\right)$.

(2) $\Rightarrow(1)$ Suppose $\Delta \in \mathcal{L}\left(k_{0}(V)\right)$. First we apply (3.9), and then $(\mathrm{G} \infty-1)$ along with (3.10) to proceed

$$
\begin{aligned}
S & \geq \sup _{i \in \mathbb{N}} \sum_{j=1}^{i} \frac{v_{m}(i)}{v_{n}(j)}\left(\begin{array}{c}
i-1 \\
j-1
\end{array}\right) \geq \sup _{i \in \mathbb{N}} \frac{v_{m}(i)}{v_{n}(1)} \sum_{j=1}^{i}\left[\frac{i^{j-1}}{(j-1) !}\left(1-\frac{1}{i}\right) \cdots\left(1-\frac{j-1}{i}\right)\right] \\
& =\sup _{i \in \mathbb{N}} \frac{v_{m}(i)}{v_{n}(1)} i^{i} \sum_{j=1}^{i}\left[\frac{1}{(j-1) ! i^{i-j+1}}\left(1-\frac{1}{i}\right) \cdots\left(1-\frac{j-1}{i}\right)\right],
\end{aligned}
$$

for a constant $S>0$. Since for any $j$,

$$
\sum_{j=1}^{i}\left[\frac{1}{(j-1) ! i^{i-j+1}}\left(1-\frac{1}{i}\right) \cdots\left(1-\frac{j-1}{i}\right)\right] \stackrel{i}{\rightarrow} 0,
$$

and $S<\infty$, one has $\sup _{i \in \mathbb{N}} i^{i} v_{m}(i)<\infty$.

Remark 3.9. Obviously, condition (U) implies nuclearity. However, the converse is not true, in general. Indeed, let $a_{n}(i):=\exp (i n)$, for $i, n \in \mathbb{N}$. Then, it is straightforward to show that $A=\left(a_{n}\right)_{n}$ is a $G_{\infty}$-matrix. Moreover, since

$$
2 \log (i)<i n \Rightarrow i^{2}<e^{i n} \quad \Rightarrow \quad e^{-i n}<\frac{1}{i^{2}}
$$


for all $i, n \in \mathbb{N}$, if we choose $m>n$ and $C>0$ as in $(\mathrm{G} \infty-2)$ we obtain

$$
\sum_{i=1}^{\infty} \frac{v_{m}(i)}{v_{n}(i)} \leq C \sum_{i=1}^{\infty} \frac{v_{n}(i)^{2}}{v_{n}(i)}=C \sum_{i=1}^{\infty} v_{n}(i) \leq C \sum_{i=1}^{\infty} \frac{1}{i^{2}}<\infty .
$$

Hence $k_{0}(V)$ is nuclear. On the other hand, we directly observe that for every $n \in \mathbb{N}, \sup _{i \in \mathbb{N}} \frac{i^{i}}{e^{i n}}=\infty$, which means the failure of condition (U).

\section{The spectrum of C In the nOn-Nuclear CASE}

In this section we give a description of the spectra $\sigma_{p t}\left(\mathrm{C} ; k_{0}(V)\right)$ and $\sigma\left(\mathrm{C} ; k_{0}(V)\right)$ when $k_{0}(V)$ is not nuclear (equivalently, $\lambda_{1}(A)$ is not nuclear). The following proposition is immediate from previous section.

Proposition 4.1. Let $\lambda_{1}(A)$ be a $G_{\infty}$-space which is Schwartz. Then, the following statements are equivalent:

(1) $k_{0}(V)$ is not nuclear.

(2) $\sigma_{p t}\left(\mathrm{C} ; k_{0}(V)\right)=\{1\}$.

(3) $0 \in \sigma\left(C ; k_{0}(V)\right)$.

Since $\mathrm{C} \in \mathcal{L}\left(k_{0}(V)\right)$, its dual $\mathrm{C}^{\prime}$ is defined and continuous on $k_{0}(V)^{\prime}$ and is given by the formula

$$
\mathrm{C}^{\prime} y:=\left(\sum_{j=i}^{\infty} \frac{y_{j}}{j}\right)_{i \in \mathbb{N}}, \quad y=\left(y_{i}\right) \in k_{0}(V)^{\prime} ;
$$

see [3, pp.774]. The following lemma is well-known. For a proof, see e.g. [15, Lemma 16].

Lemma 4.2. Let $E$ be a Fréchet space, and let $T: E \rightarrow E$ be a continuous linear operator with the dual $T^{\prime}: E^{\prime} \rightarrow E^{\prime}$. Then

$$
\sigma_{p t}\left(T^{\prime} ; E^{\prime}\right) \subset \sigma(T ; E) .
$$

For each $r>0$ we use the notation $D(r):=\left\{\lambda \in \mathbb{C}:\left|\lambda-\frac{1}{2 r}\right|<\frac{1}{2 r}\right\}$. Let $\alpha:=a(\lambda)$. Then, $\left|\lambda-\frac{1}{2 r}\right|=\frac{1}{2 r}$ if and only if $\alpha=r$.

Proposition 4.3. Let $A$ be a Köthe matrix satisfying $(G \infty-1)$. Then,

$$
\Sigma \subseteq \sigma\left(C ; k_{0}(V)\right) \subseteq \overline{D(1)}
$$

Proof. Let $\lambda \in \Sigma$, that is, there exists $s \in \mathbb{N}$ such that $\lambda=\frac{1}{s}$. Define $u^{(s)}$ by

$$
u_{i}^{(s)}:=\prod_{j=1}^{i-1}\left(1-\frac{1}{\lambda j}\right)
$$

for $1<i \leq s$ (with $u_{1}^{(s)}:=1$ ) and $u_{i}^{(s)}:=0$ for $i>s$. It is straightforward to show that $u^{(s)} \in k_{0}(V)^{\prime}$ (since $u^{(s)}$ belongs to the space of finitely supported sequences $\left.c_{00}\right)$ and $\mathrm{C}^{\prime} u^{(s)}=\frac{1}{s} u^{(s)}$, that is, $\lambda \in \sigma_{p t}\left(\mathrm{C}^{\prime}, k_{0}(V)^{\prime}\right)$. By Lemma 4.2, $\lambda \in \sigma\left(\mathrm{C} ; k_{0}(V)\right)$. This shows $\Sigma \subseteq \sigma\left(\mathrm{C} ; k_{0}(V)\right)$. By [5, Lemma 2.8] we see that $\sigma\left(\mathrm{C}_{n} ; c_{0}\left(v_{n}\right)\right) \subseteq \overline{D(1)}$ for all $n \in \mathbb{N}$, for which $\mathrm{C}_{n}: c_{0}\left(v_{n}\right) \rightarrow c_{0}\left(v_{n}\right)$ is the restriction of $\mathrm{C} \in \mathcal{L}\left(\mathbb{C}^{\mathbb{N}}\right)$. Hence $\bigcap_{s \in \mathbb{N}}\left(\bigcup_{j=s}^{\infty} \sigma\left(\mathrm{C}_{j} ; c_{0}\left(v_{j}\right)\right)\right) \subseteq \overline{D(1)}$, and so $\sigma\left(\mathrm{C} ; k_{0}(V)\right) \subseteq$ $\overline{D(1)}$ by [5, Lemma 5.5$]$. 
Proposition 4.4. Let $\lambda_{1}(A)$ be a non-nuclear, Schwartz $G_{\infty}$-space. Then

$$
\{0,1\} \cup D(1) \subseteq \sigma\left(\mathrm{C} ; k_{0}(V)\right) \subseteq \overline{D(1)} .
$$

Proof. By Proposition 4.1 and Proposition 4.3 we already know that $\Sigma_{0} \subseteq \sigma\left(\mathrm{C} ; k_{0}(V)\right) \subseteq$ $\overline{D(1)}$. So it remains to establish $D(1) \backslash \Sigma \subseteq \sigma\left(\mathrm{C} ; k_{0}(V)\right)$. Let $\lambda \in D(1) \backslash \Sigma$ and suppose that $\lambda \notin \sigma\left(\mathrm{C} ; k_{0}(V)\right)$. Then the inverse operator $(\mathrm{C}-\lambda I)^{-1}$ is continuous, equivalently, for all $n \in \mathbb{N}$ there exists $m>n$ such that $(\mathrm{C}-\lambda I)^{-1}: c_{0}\left(v_{n}\right) \rightarrow c_{0}\left(v_{m}\right)$ is continuous. Let $\beta:=a(\lambda)$ as in (3.1). Retaining the notation of Proposition 3.6 it follows that the linear map $\tilde{E}_{\lambda, n, m}: c_{0} \rightarrow c_{0}$ is continuous, where $\tilde{E}_{\lambda, n, m}=\left(\tilde{e}_{i j}^{n m}(\lambda)\right)_{i, j}$ is determined by the lower triangular matrix

$$
\tilde{e}_{i j}^{n m}(\lambda)=\frac{v_{m}(i)}{v_{n}(j)} e_{i j}(\lambda), \quad \forall i \geq 2,1 \leq j<i,
$$

and $\tilde{e}_{i j}^{n m}(\lambda)=0$, if $j \geq i$. Indeed,

$$
e_{i j}(\lambda)=\frac{1}{i \prod_{k=j}^{i}\left(1-\frac{1}{\lambda k}\right)}, \quad 1 \leq j<i,
$$

and $e_{i j}(\lambda)=0$, if $j \geq i$. Since $\tilde{E}_{\lambda, n, m} \in \mathcal{L}\left(c_{0}\right)$, by the well-known criterion [20, Theorem 4.51-C] we necessarily have $\sup _{i \in \mathbb{N}} \sum_{j=1}^{\infty} \frac{v_{m}(i)}{v_{n}(j)}\left|e_{i j}(\lambda)\right|<\infty$. By [3, pp.776] and $(\mathrm{G} \infty-1)$, there exists $C>0$ such that

$$
\sup _{i \in \mathbb{N}} \sum_{j=1}^{\infty} \frac{v_{m}(i)}{v_{n}(j)}\left|e_{i j}(\lambda)\right| \geq C \sup _{i \in \mathbb{N}} i^{\beta-1} v_{m}(i) \sum_{j=1}^{i-1} \frac{1}{j^{\beta} v_{n}(j)} \geq C \sup _{i \in \mathbb{N}} i^{\beta-1} \frac{v_{m}(i)}{v_{n}(1)} \sum_{j=1}^{i-1} \frac{1}{j^{\beta}} .
$$

Since $\beta>1$, we have

$$
\sum_{j=1}^{i-1} \frac{1}{j^{\beta}} \geq \sum_{j=1}^{i-1} \int_{j}^{j+1} \frac{1}{x^{\beta}} \mathrm{d} x=\int_{1}^{i} \frac{1}{x^{\beta}} \mathrm{d} x=\frac{1}{\beta-1}\left(1-\frac{1}{i^{\beta-1}}\right) .
$$

We have shown that for all $n \in \mathbb{N}$ there is $m>n$ such that

$$
\sup _{i \in \mathbb{N}} i^{\beta} v_{m}(i)\left(1-\frac{1}{i^{\beta-1}}\right) \leq(\beta-1) v_{n}(1) \sup _{i \in \mathbb{N}} \sum_{j=1}^{\infty} \frac{v_{m}(i)}{v_{n}(j)} \mid e_{i j}(\lambda \mid<\infty .
$$

Taking $n=1$, we get $\sup _{i \in \mathbb{N}} i^{\beta} v_{m}(i)<\infty$, for some $m \in \mathbb{N}$. By Proposition 2.9, $k_{0}(V)$ is nuclear. This is a contradiction, and $\lambda \notin \rho\left(\mathrm{C} ; k_{0}(V)\right)$.

Remark 4.5. Let $\lambda_{1}(A)$ be a $G_{\infty}$-space. Then the condition

$$
\exists n \in \mathbb{N}: \quad \sup _{i \in \mathbb{N}} \log (i) v_{n}(i)<\infty
$$

cannot be a nuclearity criterion for $k_{0}(V)$. Let $\alpha_{i}:=\log (\log (i))$, for $i \geq 3^{3}$ and consider the associated power series space $\Lambda_{\infty}^{1}(\alpha)$ of infinite type [5, Remark 3.5(ii)]. By [16, Proposition 29.6], $\Lambda_{\infty}^{1}$ is nuclear if and only if $\sup _{i \in \mathbb{N}} \alpha_{i}^{-1} \log (i)<\infty$. However, we directly observe that $\sup _{i \in \mathbb{N}} \frac{\log (i)}{\alpha_{i}}=\infty$. So, $\Lambda_{\infty}^{1}(\alpha)$ is not nuclear. It is easy to check $\Lambda_{\infty}^{1}(\alpha)$ satisfies (S) so it is Schwartz. Let $a_{n}(i):=\exp (\log (\log (i)) n)=$ $\log (i)^{n}$. Then, $\Lambda_{\infty}^{1}(\alpha)$ is isomorphic to the non-nuclear $G_{\infty}$-space $\lambda_{1}(A)$. For a fixed $n \in \mathbb{N}$, it is easy to see that $\sup _{i \in \mathbb{N}} \log (i) v_{n}(i)<\infty$. Therefore $k_{0}(V)$ satisfies condition (L).

Proposition 4.6. Let $\lambda_{1}(A)$ be a non-nuclear, Schwartz $G_{\infty}$-space. 
(1) If $k_{0}(V)$ satisfies condition (L), then $\sigma\left(\mathrm{C} ; k_{0}(V)\right)=\{0,1\} \cup D(1)$.

(2) If $k_{0}(V)$ fails condition $(\mathrm{L})$, then $\sigma\left(\mathrm{C} ; k_{0}(V)\right)=\overline{D(1)}$.

Proof. Retaining the notation of the proof of Proposition 3.6, for each $\lambda \in \mathbb{C} \backslash$ $\Sigma_{0},(\mathrm{C}-\lambda I)^{-1} \in \mathcal{L}\left(\mathbb{C}^{\mathbb{N}}\right)$ satisfies $(\mathrm{C}-\lambda I)^{-1}=D_{\lambda}-\frac{1}{\lambda^{2}} E_{\lambda}$. In the previous section we have seen that the diagonal in $D_{\lambda}$ is a bounded sequence, independent of nuclearity condition. So $(\mathrm{C}-\lambda I)^{-1}: k_{0}(V) \rightarrow k_{0}(V)$ is continuous if and only if $E_{\lambda} \in \mathcal{L}\left(k_{0}(V)\right)$. Since $k_{0}(V)=\operatorname{ind}_{n} c_{0}\left(v_{n}\right), E_{\lambda} \in \mathcal{L}\left(k_{0}(V)\right)$ if and only if for each $n \in \mathbb{N}$ there exists $m>n$ such that $E_{\lambda}: c_{0}\left(v_{n}\right) \rightarrow c_{0}\left(v_{m}\right)$ is continuous. With $\tilde{E}_{\lambda, n, m}=\left(\tilde{e}_{i j}^{n m}\right)_{i, j}$, where $\tilde{e}_{i j}^{n m}=\frac{v_{m}(i)}{v_{n}(j)} e_{n m}(\lambda)$ for $i, j \in \mathbb{N}$, it follows by the argument used in (ii) of the proof of Proposition 3.6 that $E_{\lambda}: c_{0}\left(v_{n}\right) \rightarrow c_{0}\left(v_{m}\right)$ is continuous if and only if $\tilde{E}_{\lambda, n, m}: c_{0} \rightarrow c_{0}$ is continuous. By [20, Theorem 4.51-C] it suffices to show that both (4.3) and (4.4) are satisfied:

$$
\begin{aligned}
& \lim _{i \rightarrow \infty}\left|\tilde{e}_{i j}^{n m}(\lambda)\right|=\lim _{i \rightarrow \infty} \frac{v_{m}(i)}{v_{n}(j)}\left|e_{i j}^{n m}(\lambda)\right|=0, \quad \forall j \in \mathbb{N} \\
& \sup _{i \in \mathbb{N}} \sum_{j=1}^{\infty} \frac{v_{m}(i)}{v_{n}(j)}\left|e_{i j}(\lambda)\right|=\sup _{i \in \mathbb{N}} \sum_{j=1}^{i-1} \frac{v_{m}(i)}{v_{n}(j)}\left|e_{i j}(\lambda)\right|<\infty .
\end{aligned}
$$

If $\lambda \notin\{0,1\}$ belongs to the boundary $\partial D(1)$ of $D(1)$, then $\beta:=a(\lambda)=1$ and $\lambda \notin \Sigma_{0}$. By [3, Lemma 3.3] there exist $\nu, \gamma>0$ such that

$$
\frac{\nu}{j} \leq\left|e_{i j}(\lambda)\right| \leq \frac{\gamma}{j}, \quad \forall i \in \mathbb{N}, 2 \leq j<i .
$$

Since $\lambda_{1}(A)$ is Schwartz, for any $n \in \mathbb{N}$, we find $m>n$ such that,

$$
\frac{v_{m}(i)}{v_{n}(j)}\left|e_{i j}(\lambda)\right| \leq \frac{v_{m}(i)}{v_{n}(i)} \frac{\gamma}{j} \stackrel{i}{\rightarrow} 0 .
$$

So (4.3) is satisfied. Let us recall the well-known inequality

$$
\log (i) \leq \sum_{j=1}^{i-1} \frac{1}{j} \leq 1+\log (i-1) .
$$

(1) Let us assume that there exists $n \in \mathbb{N}$ as in (L). We apply (4.5), (Go-1), (4.6) respectively, and then choose $m>n$ and $C>0$ as in $(\mathrm{G} \infty-2)$ to observe

$$
\begin{aligned}
\sup _{i \in \mathbb{N}} \sum_{j=1}^{i-1} \frac{v_{m}(i)}{v_{n}(j)}\left|e_{i j}(\lambda)\right| & \leq \gamma \sup _{i \in \mathbb{N}} v_{m}(i) \sum_{j=1}^{i-1} \frac{1}{j v_{n}(j)} \leq \gamma \sup _{i \in \mathbb{N}} \frac{v_{m}(i)}{v_{n}(i)} \sum_{j=1}^{i-1} \frac{1}{j} \\
& \leq \gamma(1+\log (i)) \frac{v_{m}(i)}{v_{n}(i)} \\
& \leq C \gamma \sup _{i \in \mathbb{N}}(1+\log (i)) v_{n}(i)<\infty .
\end{aligned}
$$

This implies (4.4) is satisfied for $\lambda \in \partial(D) \backslash\{0,1\}$, hence $\lambda \in \rho\left(\mathrm{C}, k_{0}(V)\right)$. Therefore, by Proposition 4.4, $\sigma\left(\mathrm{C} ; k_{0}(V)\right)=\{0,1\} \cup D(1)$. 
(2) Let us apply (4.5), (Gळ-1), and (4.6) respectively to obtain

$$
\begin{aligned}
\sup _{i \in \mathbb{N}} \sum_{j=1}^{i-1} \frac{v_{m}(i)}{v_{n}(j)}\left|e_{i j}(\lambda)\right| & \geq \nu \sup _{i \in \mathbb{N}} v_{m}(i) \sum_{j=1}^{i-1} \frac{1}{j v_{n}(j)} \geq \nu \sup _{i \in \mathbb{N}} \frac{v_{m}(i)}{v_{n}(1)} \sum_{j=1}^{i-1} \frac{1}{j} \\
& \geq \nu \sup _{i \in \mathbb{N}} \frac{\log (i)}{v_{n}(1)} v_{m}(i) .
\end{aligned}
$$

However, $\sup _{i \in \mathbb{N}} \log (i) v_{m}(i)=\infty$, by assumption. This means (4.4) cannot be satisfied. Hence no $\lambda \in \partial D(1) \backslash\{0,1\}$ exists which satisfies $\lambda \in \rho\left(\mathrm{C} ; k_{0}(V)\right)$, that is, $\partial D(1) \backslash\{0,1\} \subseteq \sigma\left(\mathrm{C} ; k_{0}(V)\right)$. By Proposition 4.4, we are done.

\section{MEAN ERGODICITY OF C}

Let $X$ be a Fréchet space equipped with the increasing system of seminorms $\left(p_{n}(\cdot)\right)_{n \in \mathbb{N}}$. For $S \in \mathcal{L}(X)$, the strong operator topology $\tau_{s}$ in $\mathcal{L}(X)$ is determined by the seminorms $p_{n, x}(S):=p_{n}(S x)$, for all $x \in X$ and for all $n \in \mathbb{N}$. In this case we write $\mathcal{L}_{s}(X)$. Let $\mathcal{B}(X)$ be the family of bounded subsets of $X$. Then, the uniform topology $\tau_{b}$ in $\mathcal{L}(X)$ is defined by the family of seminorms $p_{n, B}(S):=$ $\sup _{x \in B} p_{n}(S x)$, for all $n \in \mathbb{N}$ and for all $B \in \mathcal{B}(X)$, where $S \in \mathcal{L}(X)$. In this case we write $\mathcal{L}_{b}(X)$. A Fréchet space operator $T \in \mathcal{L}(X)$ is called power bounded if $\left(T^{k}\right)_{k=1}^{\infty}$ is an equicontinuous subset of $\mathcal{L}(X)$. Given $T \in \mathcal{L}(X)$, the averages $T_{[k]}:=\frac{1}{k} \sum_{j=1}^{k} T^{j}$, for $k \in \mathbb{N}$ are called the Cesàro means of $T$. The operator $T$ is said to be mean ergodic (resp., uniformly mean ergodic) if $\left(T_{[k]}\right)_{k}$ is a convergent sequence in $\mathcal{L}_{s}(X)$ (resp., in $\mathcal{L}_{b}(X)$ ).

Proposition 5.1. Let $\lambda_{1}(A)$ be a $G_{\infty}$-space which is Schwartz. Then, the Cesàro operator $\mathrm{C} \in \mathcal{L}\left(k_{0}(V)\right)$ is power bounded and uniformly mean ergodic. In particular,

$$
k_{0}(V)=\operatorname{ker}(I-\mathrm{C}) \oplus \overline{(I-\mathrm{C})\left(k_{0}(V)\right)}
$$

with $\operatorname{ker}(I-\mathrm{C})=\{\mathbf{1}\}$ and

$$
\overline{(I-\mathrm{C})\left(k_{0}(V)\right)}=\left\{x \in k_{0}(V): x_{1}=0\right\}=\overline{\operatorname{span}\left\{e_{i}\right\}_{i \geq 2}} .
$$

Proof. Let $\mathrm{C}^{k}$ be the $k$-th iterate of C. By $(\mathrm{G} \infty-1), v_{n}(i+1) \leq v_{n}(i)$, for all $i \in \mathbb{N}$. Then, by [3, Corollary 2.3(i)] $\mathrm{C} \in \mathcal{L}\left(c_{0}\left(v_{n}\right)\right)$ and $q_{n}(\mathrm{C} x) \leq q_{n}(x)$, for all $x \in c_{0}\left(v_{n}\right)$. By (1.4), for every $n \in \mathbb{N}$ we have $q_{n}\left(\mathrm{C}^{k} x\right) \leq q_{n}(x)$, for all $x \in c_{0}\left(v_{n}\right)$, and $k \in \mathbb{N}$. [5, Lemma 5.4] implies $\mathrm{C}$ is power bounded in $k_{0}(V)$. It follows by Propositions 2.4 and 2.8 in [1] that $\mathrm{C}$ is uniformly mean ergodic in $k_{0}(V)$ and hence (5.1) is also satisfied.

Proposition 5.2. Let $\lambda_{1}(A)$ be a Schwartz $G_{\infty}$-space satisfying condition (L). Then the range $(I-\mathrm{C})^{j}\left(k_{0}(V)\right)$ is a closed subspace of $k_{0}(V)$ for each $j \in \mathbb{N}$.

Proof. First we consider the case $j=1$. Set $X(V):=\left\{x \in k_{0}(V): x_{1}=0\right\}$. We claim that $(I-\mathrm{C})\left(k_{0}(V)\right)=(I-\mathrm{C})(X(V))$. We proceed as in the proof of the analogous result in [5, Proposition 4.3]. By condition (I), there exists $n_{0} \in \mathbb{N}$ such

that $v_{n}(i) \stackrel{i}{\rightarrow} 0$, for all $n \geq n_{0}$. Since $k_{0}(V)$ is an inductive limit of increasing Banach spaces, we can clearly assume that $v_{n}(i) \stackrel{i}{\rightarrow} 0$, for all $n \in \mathbb{N}$. So each $v_{k}$ is strictly positive and decreasing with $v_{n} \in c_{0}$ and hence $\overline{(I-\mathrm{C})\left(c_{0}\left(v_{n}\right)\right)}=$ $\left\{x \in c_{0}\left(v_{n}\right): x_{1}=0\right\}=: X_{n}$ and $(I-\mathrm{C})\left(X_{k}\right)=(I-\mathrm{C})\left(c_{0}\left(v_{n}\right)\right)$ by [3, Lemma 
4.1 and Lemma 4.5]. If $x \in X(V)$, then $x \in X_{n}$ for some $n \in \mathbb{N}$ and $(I-\mathrm{C}) x \in$ $(I-\mathrm{C})\left(X_{n}\right)=(I-\mathrm{C})\left(c_{0}\left(v_{n}\right)\right) \subseteq(I-\mathrm{C})\left(k_{0}(V)\right)$. That fulfills one inclusion. Now let $x \in k_{0}(V)$. Then $x \in c_{0}\left(v_{n}\right)$ for some $n \in \mathbb{N}$ and hence $(I-\mathrm{C}) x \in(I-\mathrm{C})\left(c_{0}\left(v_{n}\right)\right)=$ $(I-\mathrm{C})\left(X_{n}\right) \subseteq(I-\mathrm{C})(X(V))$. Hence $(I-\mathrm{C})\left(k_{0}(V)\right)=(I-\mathrm{C})(X(V))$. To prove that $(I-\mathrm{C})\left(k_{0}(V)\right)$ is closed in $k_{0}(V)$, it suffices to show that $(I-\mathrm{C}) \in \mathcal{L}(X(V))$ is surjective: if $(I-\mathrm{C})(X(V))=X(V)$, then $(I-\mathrm{C})\left(k_{0}(V)\right)=X(V)$ and hence $(I-\mathrm{C})\left(k_{0}(V)\right)$ is closed in $k_{0}(V)$. By [8, Lemma 6.3.1], $(X(V), \tau)=\operatorname{ind}_{n} X_{n}$, where $\tau$ is the relative topology in $X(V)$ induced from $k_{0}(V)$. If we set $\tilde{v}_{n}(i):=v_{n}(i+1)$, for all $i, n \in \mathbb{N}$, then we have the topological isomorphism $X(V) \simeq E:=\operatorname{ind}_{n} c_{0}\left(\tilde{v}_{n}\right)$ by the left shift operator $S: X(V) \rightarrow E$ which is a surjective isomorphism at each step $S: X_{n} \rightarrow c_{0}\left(v_{n}\right)$. Let $T:=\left.S \circ(I-\mathrm{C})\right|_{X(V)} \circ S^{-1} \in \mathcal{L}(E)$. We now prove that $A$ is bijective with $B:=T^{-1} \in \mathcal{L}(E)$. It is straightforward to see $T: \mathbb{C}^{\mathbb{N}} \rightarrow \mathbb{C}^{\mathbb{N}}$ is bijective and its inverse $B$ is given by the lower triangular matrix $\left(b_{i j}\right)$ whose entries are $\frac{1}{j}$ for $1 \leq j<i, \frac{i+1}{i}$ for $j=i$, and 0 for $j>i$. To show that $B$ is still the inverse of $T$ when acting on $E$, we must prove $B \in \mathcal{L}(E)$, equivalently, for each $n \in \mathbb{N}$ there exists $m>n$ such that $\phi_{m} \circ B \circ \phi_{n}^{-1} \in \mathcal{L}\left(c_{0}\right)$, where the surjective isometry $\phi_{k}: c_{0}\left(\tilde{v}_{k}\right) \rightarrow c_{0}$ is given by $\phi_{k} x=\left(v_{k}(i+1) x_{i}\right)$ for every $x \in c_{0}\left(\tilde{v}_{k}\right)$. The lower triangular matrix corresponding to $\phi_{m} \circ B \circ \phi_{n}^{-1}$ is given by $d_{i j}:=\left(\frac{v_{m}(i+1)}{v_{n}(j+1)} b_{i j}\right)$, for all $i, j \in \mathbb{N}$. For every $j, \lim _{i \rightarrow \infty} \frac{v_{m}(i+1)}{v_{n}(j+1)} b_{i j}=\frac{1}{j v_{n}(j+1)} \lim _{i \rightarrow \infty} v_{m}(i+1)=0$. We make use of $(\mathrm{G} \infty-1)$ and (4.6), respectively, then pick $m>n$ and $C>0$ as in $(\mathrm{G} \infty-2)$, and then finally use condition (L) to observe

$$
\begin{aligned}
\sum_{j=1}^{\infty} \frac{v_{m}(i+1)}{v_{n}(j+1)} b_{i j} & =\frac{i+1}{i} \frac{v_{m}(i+1)}{v_{n}(i+1)} \sum_{j=1}^{i-1} \frac{1}{j v_{n}(j+1)} \leq \frac{v_{m}(i+1)}{v_{n}(i+1)}\left(\frac{i+1}{i}+\sum_{j=1}^{i-1} \frac{1}{j}\right) \\
& \leq \frac{v_{m}(i+1)}{v_{n}(i+1)}\left(\frac{i+1}{i}+(1+\log (i-1))\right) \\
& \leq C(3+\log (i)) v_{n}(i+1)<\infty .
\end{aligned}
$$

Hence both the conditions (i) and (ii) of [3, Lemma 2.1] hold. Then, $\phi_{m} \circ B \circ \phi_{n}^{-1} \in$ $\mathcal{L}\left(c_{0}\right)$ and hence $(I-\mathrm{C})\left(k_{0}(V)\right)$ is closed. Since $(I-\mathrm{C})\left(k_{0}(V)\right)$ is closed, (5.1) implies $k_{0}(V)=\operatorname{ker}((I-\mathrm{C})) \oplus(I-\mathrm{C})\left(k_{0}(V)\right)$. The proof of $(2) \Rightarrow(5)$ in [2, Remark 3.6] implies that $(I-\mathrm{C})^{j}\left(k_{0}(V)\right)$ is closed in $k_{0}(V)$, for all $j \in \mathbb{N}$.

Let $X$ be a separable Fréchet space. Then the operator $T \in \mathcal{L}(X)$ is called hypercyclic if there exists $x \in X$ such that the orbit $\left\{T^{k} x: k \in \mathbb{N}_{0}\right\}$ is dense in $X$. If, for some $z \in X$, the projective orbit $\left\{\lambda T^{k} z: \lambda \in \mathbb{C}, k \in \mathbb{N}_{0}\right\}$ is dense in $X$, then $T$ is called supercyclic. Clearly, if $\mathrm{C}$ is hypercyclic then $\mathrm{C}$ is supercyclic.

Proposition 5.3. Let $A$ be a Köthe matrix. Then $\mathrm{C} \in \mathcal{L}\left(k_{0}(V)\right)$ is not supercyclic, and hence not hypercyclic either.

Proof. Follows from [4, Proposition 4.3] and [5, Proposition 4.4].

\section{ACKNOWLEDGEMENTS}

The author wishes to thank Prof. José Bonet for useful suggestions and discussions. 


\section{REFERENCES}

[1] A. Albanese, J. Bonet, and W. Ricker. Mean ergodic operators in Fréchet spaces. Ann. Acad. Sci. Fenn. Math., 34:401-436, 2009.

[2] A. Albanese, J. Bonet, and W. Ricker. Convergence of arithmetic means of operators in Fréchet spaces. J. Math. Anal. Appl., 401:160-173, 2013.

[3] A. Albanese, J. Bonet, and W. Ricker. Mean ergodicity and spectrum of the Cesàro operator on weighted $c_{0}$ spaces. Positivity, 20:761-803, 2016.

[4] A. Albanese, J. Bonet, and W. Ricker. The Cesàro operator in the Fréchet spaces $\ell^{p+}$ and $L^{p-}$. Glasg. Math. J., 59(2):273-287, 2017.

[5] A. Albanese, J. Bonet, and W. Ricker. The Cesàro operator on duals of power series spaces of infinite type. J. Operator Theory, 79(2):373-402, 2018.

[6] K. D. Bierstedt. An introduction to locally convex inductive limits. In H. Hogbe-Nlend, editor, Functional Analysis and its Applications, pages 35133, Singapore, 1988. World Scientific.

[7] K. D. Bierstedt, R. Meise, and W. H. Summers. Köthe sets and Köthe sequence spaces. In J. A. Barroso, editor, Functional Analysis, Holomorphy and Approximation Theory, volume 71 of North-Holland Math. Studies, pages 27-91, New York, 1982. North Holland.

[8] P. Pérez Carreras and J. Bonet. Barrelled Locally Convex Spaces, volume 131 of North Holland Math. Studies. North Holland, Amsterdam, 1987.

[9] M. M. Dragilev. Bases in Köthe spaces (Russian). Rostov: Izdatel'sto Rostovskogo Universiteta, Rostov-on-Don, 1983.

[10] R. E. Edwards. Functional Analysis. Theory and Applications. Holt, Rinehart and Winston, New York Chicago San Francisco, 1965.

[11] M. Kocatepe. On Dragilev spaces and the functor Ext. Arch. Math. (Basel), 44:438-445, 1985.

[12] M. Kocatepe. Classification of Dragilev spaces of types-1 and 0. Math. Balkanica, 2(2-3):266-275, 1988.

[13] M. Kocatepe and Z. Nurlu. Some special Köthe spaces. In T. Terziog̃lu, editor, Advances in the Theory of Fréchet Spaces, volume 287 of Series C: Mathematical and Physical Sciences, pages 269-296, Dordrecht, 1989. Kluwer.

[14] G. Köthe. Topological Vector Spaces I. Springer, Berlin Heidelberg, 1969.

[15] E. Kizgut. The Cesàro operator on smooth sequence spaces of finite type. Rev. R. Acad. Cienc. Exactas Fís. Nat. Ser. A Math. RACSAM, 2018.

[16] R. Meise and D. Vogt. Introduction to Functional Analysis. Number 2 in Oxford Graduate Texts in Mathematics. Clarendon Press, Oxford, 1997.

[17] A. Pietsch. Nuclear Locally Convex Spaces, volume 66 of Ergebnisse der Mathematik und ihrer Grenzgebeite. Springer-Verlag, Berlin, 1972.

[18] M. S. Ramanujan and T. Terziog̃lu. Subspaces of smooth sequence spaces. Studia Math., 65:299-312, 1979.

[19] J. B. Reade. On the spectrum of the Cesàro operator. Bull. Lond. Math. Soc., 17:263-267, 1985.

[20] A. E. Taylor. Introduction to Functional Analysis. Wiley, New York, 1958.

[21] T. Terziog̃lu. Die diametral Dimension von lokalkonvexen Räumen. Collect. Math., 20:49-99, 1969.

[22] T. Terziog̃lu. Smooth sequence spaces and associated nuclearity. Proc. Amer. Math. Soc., 37(2):497-504, 1973. 
[23] T. Terziog̃lu. Stability of smooth sequence spaces. J. Reine Angew. Math., 276:184-189, 1975.

Instituto Universitario de Matemática Pura y Aplicada, Universitat Politècnica de València, E-46071 Valencia, Spain

E-mail address: erkiz@upv.es 